\title{
Efficacy of gelatin sponge impregnated with ropivacaine on postoperative pain after transforaminal lumbar interbody fusion: a comparative study
}

Shanxi Wang, Bo Wang, Xiaojun Yu, Tian Ma, Mubotu C. Ntambale, Jiyuan Yan, Qing Ding, Ruizhuo Zhang, Hua Wu ${ }^{*}$ and Chaoxu Liu*

\begin{abstract}
Background: The purpose of this study was to investigate the efficacy of gelatin sponge impregnated with ropivacaine on postoperative pain after transforaminal lumbar interbody fusion (TLIF) in patients with lumbar degenerative diseases.
\end{abstract}

Methods: We retrospectively reviewed patients who underwent TLIF in our department between August 2018 and January 2020. Patients were divided to ropivacaine group and saline group. A ropivacaine group whom received gelatin sponge impregnated with ropivacaine during operation, and a saline group whom were intraoperatively administered by gelatin sponge impregnated with saline. The two groups were compared in reference to postoperative hospital stay, postoperative complications and visual analog scale (VAS) scores. The consumption of postoperative diclofenac sodium suppository use was also recorded. The Oswestry Disability Index (ODI) scores and Japanese Orthopedic Association (JOA) scores were used for functional evaluation at 1 year postoperatively.

Result: A total of 127 patients were evaluated in this retrospective study. The mean postoperative hospital stay in the ropivacaine group was significantly lower than saline group. The VAS score was significantly lower in patients receiving gelatin sponge impregnated with ropivacaine as compared with patients in saline group on postoperative day 1, 2, 3 and 4 . The number of patients who need the administration of diclofenac sodium suppository and the mean consumption of postoperative diclofenac sodium suppository was significantly lower in the ropivacaine group as compared with saline group.

Conclusion: The application of gelatin sponge impregnated with ropivacaine around the nerve root in patients undergoing TLIF can effectively control the postoperative pain and reduce postoperative hospital stay.

Keywords: Ropivacaine, Gelatin sponge, Postoperative pain, Transforaminal lumbar interbody fusion, Lumbar degenerative diseases

\footnotetext{
* Correspondence: wuhua@hust.edu.cn; chaoxuliu@hotmail.com Department of Orthopedics, Tongji Hospital, Tongji Medical College, Huazhong University of Science and Technology, Jiefang Avenue 1095, Wuhan 430030, People's Republic of China
}

C The Author(s). 2021 Open Access This article is licensed under a Creative Commons Attribution 4.0 International License, which permits use, sharing, adaptation, distribution and reproduction in any medium or format, as long as you give appropriate credit to the original author(s) and the source, provide a link to the Creative Commons licence, and indicate if changes were made. The images or other third party material in this article are included in the article's Creative Commons licence, unless indicated otherwise in a credit line to the material. If material is not included in the article's Creative Commons licence and your intended use is not permitted by statutory regulation or exceeds the permitted use, you will need to obtain permission directly from the copyright holder. To view a copy of this licence, visit http://creativecommons.org/licenses/by/4.0/ The Creative Commons Public Domain Dedication waiver (http://creativecommons.org/publicdomain/zero/1.0/) applies to the data made available in this article, unless otherwise stated in a credit line to the data. 


\section{Background}

Transforaminal lumbar interbody fusion (TLIF) is a highly effective intervention for treating severe lumbar degenerative diseases, such as lumbar disc herniation, lumbar spondylolisthesis and lumbar spinal stenosis [1]. However, because of the spinal structure damage and nerve root traction during operation, moderate-to-severe post-surgical pain after TLIF is frequently encountered in the early postoperative period, which often leads to limitations in patient recovery and prolonged hospital stay $[2,3]$. Therefore, an effective method adopted by surgeons or anesthetists is essential to relief postoperative pain and improve the comfort of patients after operation.

Ropivacaine is a long-acting amide local anesthetic with an onset time of about $10 \mathrm{~min}$ and a duration of 4 to $5 \mathrm{~h}$. It is characterized by blocking of the sensory nerve fibers superior to motor nerve fibers, and it can cause separation block between sensory nerve fibers and motor nerve fibers even in a low concentration $(0.2 \%)$ [4]. As a results, ropivacaine is increasingly used in postoperative rehabilitation of surgical patients, which can effectively block the sensory nerve for analgesia without affecting the motor function of patients [5].

In this paper, we investigated the efficacy of gelatin sponge impregnated with ropivacaine on postoperative pain after transforaminal lumbar interbody fusion in patients with lumbar degenerative diseases, so as to provide reference for clinical treatment.

\section{Patients and methods \\ Study sample}

After obtaining approval from our institutional review board, we retrospectively reviewed patients who underwent TLIF in our department between August 2018 and January 2020. The inclusion criteria were as follows: (a) lumbar degenerative diseases, including lumbar disc herniation, lumbar spondylolisthesis and lumbar spinal stenosis, (b) primary lumbar surgery, (c) with at least 1 year of follow-up. Exclusion criteria included other spinal pathology (tumor, trauma, congenital or infection) and known allergy to the ropivacaine. Based on whether they were administered by gelatin sponge impregnated with ropivacaine or gelatin sponge impregnated with saline during operation, patients were divided into ropivacaine group or saline group.

\section{Surgical technique}

All surgeries were performed under general anesthesia by one surgical team consisting of two senior orthopedic surgeons. The patient was placed prone on a radiolucent table, a posterior midline lumbar incision was performed. After exposure of bilateral lamina and facet joints, unilateral facetectomy and partial laminectomy were done to expose the intervertebral disc and achieve adequate posterior decompression. The reamer was used to remove disc tissue, and then a suitable size cage filled with the autologous bone graft was inserted into the intervertebral space. Subsequently, bilateral pedicle screws and titanium rods were installed and axially compressed to restore the lordosis, while maintaining the recovered disc height. For patients in ropivacaine group, 2 pieces of gelatin sponge $(6 \mathrm{~cm} \times 2 \mathrm{~cm} \times 0.5 \mathrm{~cm})$ impregnated with $0.75 \%$ ropivacaine were used to gently covered the surface of dura mater and nerve root. In saline group, patients received the gelatin sponge impregnated with $0.9 \%$ saline covered on the surface of dura mater and nerve root (Fig. 1). Finally, the incision was closed after placement of a drainage tube.

\section{Postoperative management and aftercare}

The prophylactic intravenous antibiotics were administered after operation for $24 \mathrm{~h}$ to prevent infection. Enoxaparin $(0.4 \mathrm{~mL})$ was administered subcutaneously every $24 \mathrm{~h}$ until discharge to prevent deep venous thrombosis (DVT). The drainage was maintained for $24-48 \mathrm{~h}$ and then was removed. Parecoxib $40 \mathrm{mg}$ every $12 \mathrm{~h}$ intramuscularly to control postoperative pain, and two diclofenac sodium suppositories $(25 \mathrm{mg}$ ) were used when the patients complained pain with the visual analog scale (VAS) score [6] more than 6. No patient-controlled analgesia (PCA) pump was used in all patients. Limb functional exercises were encouraged after recovery from anesthesia, and out-of-bed activity as tolerated were encouraged after surgery.

\section{Outcome measurements}

Patient demographic included age, gender, body mass index (BMI), diagnosis, American Society of Anesthesiologists (ASA) physical status, fusion level, fusion site, preoperative functional scores, operative time, blood loss and postoperative drainage volume were collected and compared. Clinical parameters included postoperative hospital stay and postoperative complications. Pain level was assessed using the VAS score preoperatively and on postoperative days $1,2,3,4,5$ (POD 1, 2, 3, 4, 5). The rescue use of diclofenac sodium suppository was also recorded. After discharge, the patients would be followed at 1, 2, 3, 6 and 12 months and then after annually postoperatively in clinic. The Oswestry Disability Index (ODI) scores [7], and Japanese Orthopedic Association (JOA) scores [8] were used for functional evaluation at 1 year postoperatively.

\section{Statistical analysis}

All data management and statistical analysis were performed with Statistical Package for the Social Sciences (SPSS 20.0, IBM, New York City, USA). Categorical data 

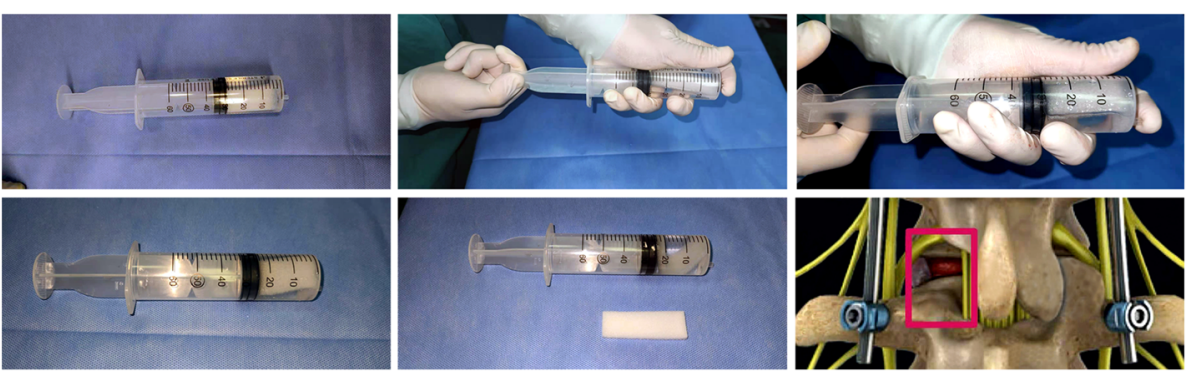

Fig. 1 Operation steps of gelatin sponge impregnated with ropivacaine. a The gelatin sponge was immersed in a syringe filled with ropivacaine, and the gas in the syringe was evacuated. $\mathbf{b}$ Plug the outlet of the syringe with a finger while pulling back the piston repeatedly with the other hand to create a negative pressure in the syringe. $\mathbf{c}$ The residual gas in the gelatin sponge was sucked out by negative pressure and formed bubbles in the syringe. $\mathbf{d}$-e Remove the bubbles from the syringe, the volume of ropivacaine in the syringe was significantly decreased compared to before, which proved that the gelatin sponge further absorbed ropivacaine. $\mathbf{f}$ The location of the intraoperatively gelatin sponge

were tabulated with frequencies or percentages, and continuous data were expressed as the mean \pm standard deviation (SD). Normality was tested using the Kolmogorov-Smirnov test. Independent t-tests were used for normally distributed continuous data and the
Mann-Whitney test was used to compare abnormally distributed continuous data between two groups. Chisquare test or Fisher exact test was used to analyze the categorical variables. The level of significance was set at $p<0.05$.

Table 1 Baseline characteristics of the study population

\begin{tabular}{|c|c|c|c|}
\hline & Ropivacaine $(n=62)$ & Saline $(n=65)$ & $P$ value \\
\hline Age (years) & $53.63 \pm 8.81$ & $53.91 \pm 11.60$ & 0.879 \\
\hline Gender (male/female) & $31 / 31$ & $35 / 30$ & 0.724 \\
\hline $\mathrm{BMI}\left(\mathrm{kg} / \mathrm{m}^{2}\right)$ & $23.76 \pm 2.72$ & $23.02 \pm 2.68$ & 0.128 \\
\hline Diagnosis & & & 0.122 \\
\hline LDH & 41 & 39 & \\
\hline LS & 14 & 10 & \\
\hline LSS & 7 & 16 & \\
\hline ASA status (I/I/IIII) & 10/51/1 & $12 / 48 / 5$ & 0.264 \\
\hline Fusion segments (number) & $1.68 \pm 0.76$ & $1.48 \pm 0.66$ & 0.116 \\
\hline Fusion level (s) & & & 0.352 \\
\hline 1 Level & 30 & 40 & \\
\hline 2 Level & 23 & 19 & \\
\hline 3 Level & 8 & 6 & \\
\hline 4 Level & 1 & 0 & \\
\hline Fusion site (s) & & & 0.781 \\
\hline L2-L3 & 1 & 3 & \\
\hline L3-L4 & 14 & 13 & \\
\hline L4-L5 & 53 & 46 & \\
\hline L5-S1 & 36 & 34 & \\
\hline Preoperative ODI scores & $62.93 \pm 15.92$ & $62.68 \pm 17.70$ & 0.931 \\
\hline Preoperative JOA scores & $6.85 \pm 3.69$ & $7.06 \pm 2.90$ & 0.725 \\
\hline Preoperative VAS scores & $6.76 \pm 1.13$ & $6.91 \pm 1.13$ & 0.456 \\
\hline Operative time (min) & $208.08 \pm 53.23$ & $204.43 \pm 54.23$ & 0.703 \\
\hline Blood loss (ml) & $314.52 \pm 169.79$ & $287.25 \pm 188.34$ & 0.394 \\
\hline Drainage volume (ml) & $329.52 \pm 229.81$ & $383.75 \pm 273.64$ & 0.230 \\
\hline
\end{tabular}




\section{Results}

Baseline characteristics

A total of 127 patients were evaluated in this retrospective study, which included 62 patients in ropivacaine group and 65 patients in saline group. Baseline characteristics of the patients in both groups were summarized and comparable in Table 1. There was no statistically significant differences between the two groups in terms of age, gender, diagnosis, ASA physical status, fusion level, fusion site, preoperative functional scores, operative time, blood loss and postoperative drainage volume.

\section{Clinical outcomes}

The mean postoperative hospital stay was $4.97 \pm 1.43$ days for the ropivacaine group and $6.23 \pm 1.44$ days for the saline group $(P<0.001)$. Pain scores on POD $1,2,3$ and 4 were significantly lower for ropivacaine group compared to saline group $(2.40 \pm 0.86$ VS. $3.18 \pm 1.01$, $P<0.001 ; 2.79 \pm 0.94$ VS. $3.85 \pm 1.11, P<0.001 ; 2.48 \pm$ 0.90 VS. $2.95 \pm 0.82, P=0.003 ; 2.24 \pm 0.82$ VS. $2.58 \pm$ $0.79, P=0.018$, respectively), no statistical difference was identified between two groups on POD 5. In ropivacaine group, 19 patients required the administration of diclofenac sodium suppository, and the mean consumption of diclofenac sodium suppository was $15.32 \pm 28.76 \mathrm{mg}$. In saline group, the number of patients who need analgesic rescue was 34, and the mean consumption of diclofenac sodium suppository was $28.46 \pm 34.20 \mathrm{mg}$. Fewer patients required diclofenac sodium suppository in ropivacaine group than saline group and the difference was statistically significant. Lower diclofenac sodium suppository consumption was found in ropivacaine group than saline group, and the difference also has statistically significant. Delayed wound healing was found in one patient in ropivacaine group and three patients in saline group, no postoperative nausea and vomiting (PONV) or DVT was found in neither group. There was no statistically significant difference between two groups in the incidence of postoperative complications. (Table 2).

At one year after surgery, the mean ODI scores of ropivacaine group was $10.81 \pm 5.52$, while the mean ODI scores of saline group was $11.97 \pm 6.26$. The average JOA scores of ropivacaine group and saline group were $27.06 \pm 1.33$ and $26.71 \pm 1.73$, respectively. There were no statistically significant differences between two groups in the ODI scores and JOA scores at one year postoperatively. (Table 3 ).

\section{Discussion}

The lower back pain is a leading reason of disability worldwide, which often caused by lumbar degenerative diseases, such as lumbar disc herniation, lumbar spondylolisthesis and lumbar spinal stenosis [9]. For patients who have failed non-surgical treatment, lumbar fusion is an effective option, which not only relieves pain, but also improves the life quality of patients $[10,11]$. According to the surgical approach, lumbar fusion can be divided into different types, the most common are anterior lumbar interbody fusion (ALIF), lateral lumbar interbody fusion (LLIF), posterior lumbar interbody fusion (PLIF) and TLIF [12].

Table 2 Comparison of postoperative hospital stay, VAS scores, requirement of diclofenac sodium suppositories and postoperative complications between the two groups

\begin{tabular}{|c|c|c|c|}
\hline & Ropivacaine $(n=62)$ & Saline $(n=65)$ & $P$ value \\
\hline Postoperative hospital stay (days) & $4.97 \pm 1.43$ & $6.23 \pm 1.44$ & $<0.001$ \\
\hline \multicolumn{4}{|l|}{ VAS scores } \\
\hline POD1 & $2.40 \pm 0.86$ & $3.18 \pm 1.01$ & $<0.001$ \\
\hline POD2 & $2.79 \pm 0.94$ & $3.85 \pm 1.11$ & $<0.001$ \\
\hline POD3 & $2.48 \pm 0.90$ & $2.95 \pm 0.82$ & 0.003 \\
\hline POD4 & $2.24 \pm 0.82$ & $2.58 \pm 0.79$ & 0.018 \\
\hline POD5 & $2.10 \pm 0.82$ & $2.23 \pm 0.64$ & 0.254 \\
\hline \multicolumn{4}{|c|}{ Diclofenac sodium suppository } \\
\hline \multicolumn{4}{|l|}{ Number (n) } \\
\hline Dose (mg) & $15.32 \pm 28.76$ & $28.46 \pm 34.20$ & 0.010 \\
\hline \multicolumn{4}{|l|}{ Complications } \\
\hline Delayed wound healing & 1 & 3 & 0.619 \\
\hline PONV & 0 & 0 & - \\
\hline DVT & 0 & 0 & - \\
\hline
\end{tabular}

VAS Visual analog scale, POD Postoperative day, PONV Postoperative nausea and vomiting, DVT Deep venous thrombosis 
Table 3 Comparison of ODI scores and JOA scores at 1 year postoperatively between two groups

\begin{tabular}{llll}
\hline & Ropivacaine $(\mathbf{n}=\mathbf{6 2})$ & Saline $(\mathbf{n}=\mathbf{6 5})$ & $\boldsymbol{P}$ value \\
\hline ODI scores & $10.81 \pm 5.52$ & $11.97 \pm 6.26$ & 0.270 \\
JOA scores & $27.06 \pm 1.33$ & $26.71 \pm 1.73$ & 0.139 \\
\hline
\end{tabular}

ODI Oswestry Disability Index, JOA Japanese Orthopaedic Association

TLIF was first described by Harms and developed as a modification of PLIF [13]. Compared to other approaches, it can directly access to the intervertebral foramen area, with little damage to the structural integrity of spinal [14-16]. There are also evidences show that TLIF can reduce the risk of dural tears and nerve root injury although has a lower rate of postoperative complications and better functional recovery $[10,15]$. As a result, TLIF has become a well-established and prevalent surgical approach for degenerative lumbar diseases [14, 15]. However, there are also disadvantages of TLIF, one of the most major challenges is the severe postoperative pain related to the extensive muscle dissection and nerve root stimulation during operation [10]. As uncontrolled postoperative pain is directly associated to longer hospital stays, increased costs, delayed recovery and more complications, the management of postoperative pain is highly imperative for patients undergoing TLIF [17].

Although opioids or non-steroidal anti-inflammatory drugs play an important role in the control of postoperative pain for spinal patients, the side effects such as PONV, pruritus, respiratory depression or peptic ulcer still limited their use $[18,19]$. Besides, excessive use of opioids can contribute to long-term opioids dependence and abuse [20]. Therefore, the control of postoperative pain is essential to curb the overuse of analgesics and related adverse outcomes. Prasartritha et al. [21] reported that epidural infusion analgesia is safe and effective for controlling postoperative pain in spinal surgery. However, the technique is sometimes very dangerous because of the possible penetration of duramater, which can injure spinal cord and cause total spinal subarachnoid anesthesia [22]. Local infiltration analgesia may be an another alternative method to control postoperative pain in spinal surgery. Tomov et al. [23] investigated the efficacy of subcutaneous infiltration of liposomal bupivacaine on postoperative pain management and narcotic use following TLIF. Although the result showed that the application of local infiltration can significantly reduce postoperative pain and the consumption of postoperative analgesic, it however does not significantly reduce the length of hospital stay.

Patients undergoing spinal surgery may experience intense pain in the early postoperative period, the use of gelatin sponge can slowly release ropivacaine around the nerve root so as to extend the postoperative analgesia time [2, 24]. In most studies, ropivacaine was injected into gelatin sponge by syringe $[8,25]$. However, because of the residual gas in the gelatin sponge, it is difficult to make the gelatin sponge completely absorb ropivacaine in this method. We created a negative pressure in the syringe, which can effectively exhaust the residual gas in the gelatin sponge, so that the gelatin sponge can absorb enough ropivacaine, thereby increasing the release time of ropivacaine and prolonging the postoperative analgesia effect. Our results showed that the application of gelatin sponge impregnated with ropivacaine could significantly reduce the postoperative pain on POD 1,2, 3 and 4, especially on POD 1 and 2, the VAS scores of ropivacaine group was markedly lower than saline group. Fewer patients in ropivacaine group need the administration of diclofenac sodium suppository, and the mean consumption of diclofenac sodium suppository was also less in ropivacaine group. Besides, our research also suggested that the application of gelatin sponge impregnated with ropivacaine during operation can effectively shorten the postoperative hospital stay, which may be due to the limitation of postoperative pain. The control of postoperative pain is conducive to the recovery of postoperative function in spinal patients, and the early out-of-bed activity is beneficial to the prevention of pulmonary complications and deep venous thrombosis. In addition, a shorter length of postoperative hospital stay means that the costs of hospitalization can be saved.

There are several limitations to our study. One of the limitations is that this was a retrospective study. A randomized controlled study is needed to further investigated the efficacy of gelatin sponge impregnated with ropivacaine on postoperative pain after transforaminal lumbar interbody fusion in patients with lumbar degenerative diseases. Secondly, since all surgeries in this study were performed by one surgical team including two senior orthopedic surgeons at a single center, multicenter research is needed to further verify our conclusions. Furthermore, further study is required to compare the efficacy for gelatin sponge impregnated with ropivacaine and other analgesic strategies on postoperative pain in patients treated with TLIF.

\section{Conclusions}

Our study demonstrated that the application of gelatin sponge impregnated with ropivacaine in patients undergoing TLIF can effectively control the immediate acute postoperative pain and reduce postoperative hospital stay.

\section{Acknowledgments \\ We wish to thank all of those who generously agreed to be interviewed for this research.}

\section{Authors' contributions}

Shanxi Wang and Bo Wang contributed equally to this work. Chaoxu Liu and Hua Wu conceived and designed this study; Shanxi Wang, Bo Wang, Xiaojun 
Yu, Tian Ma, Ruizhuo Zhang collected the data; Mubotu C. Ntambale assisted in language editing; Shanxi Wang, Bo Wang, Jiyuan Yan and Qing Ding performed the statistical analysis; Shanxi Wang wrote the manuscript; Chaoxu Liu revised this manuscript. All authors reviewed the final manuscript. All authors agree to be accountable for all aspects of the work.

\section{Funding}

This work was supported by National Natural Science Foundation of China (No.51537004 and No. 51877097).

\section{Availability of data and materials}

The data sets generated and/or analysed during the current study are not publicly available due to limitations of ethical approval involving the patient data and anonymity but are available from the corresponding author on reasonable request.

\section{Declarations}

\section{Ethics approval and consent to participate}

The study was approved by the ethical committee of Tongji Hospital, Tongji Medical College, Huazhong University of Science and Technology. All procedures performed in studies involving human participants were in accordance with the ethical standards of institutional and/or national research committee and within the 1964 Helsinki Declaration and its later amendments or comparable ethical standards. Written informed consent was obtained from all patients included in this study.

\section{Consent for publication}

Not applicable.

\section{Competing interests}

The authors declare that they have no conflict of interest.

Received: 13 May 2021 Accepted: 23 July 2021

Published online: 06 August 2021

\section{References}

1. Rosenberg WS, Mummaneni PV. Transforaminal lumbar interbody fusion: technique, complications, and early results. Neurosurgery. 2001;48(3):574-5. https://doi.org/10.1097/00006123-200103000-00022.

2. Ghabach MB, Mhanna NE, Abou AEM, et al. Comparison of effects of hemostatic gelatin sponge impregnated with Ropivacaine versus Normal saline applied on the transverse process of the operated vertebrae on postoperative pain in patients undergoing spinal instrumentation surgery: a randomized clinical trial. World Neurosurg. 2019;128:e1126-30. https://doi. org/10.1016/j.wneu.2019.05.101.

3. Aljabi Y, Aljabi Y. Effect of epidural methylprednisolone on post-operative pain and length of hospital stay in patients undergoing lumbar microdiscectomy. Surg J Royal Colleges Surg Edinburgh Ireland. 2015;13(5): 245-9. https://doi.org/10.1016/.jsurge.2014.03.012.

4. Markham A, Faulds D. Ropivacaine. Drugs. 1996;52(3):429-49. https://doi. org/10.2165/00003495-199652030-00012.

5. Axelsson $\mathrm{K}$, Johanzon E, Essving P, Weckstrom J, Ekback G. Postoperative extradural analgesia with morphine and ropivacaine. A double-blind comparison between placebo and ropivacaine $10 \mathrm{mg} / \mathrm{h}$ or $16 \mathrm{mg} / \mathrm{h}$. Acta Anaesth Scand. 2005;49(8):1191-9. https://doi.org/10.1111/j.1399-6576.2005. 00715.x.

6. Huskisson EC. Measurement of pain. LANCET. 1997;2(7889):1127-31. https:// doi.org/10.1016/50140-6736(74)90884-8.

7. Fairbank J, Pynsent PB. The Oswestry disability index. SPINE. 2000;25(22): 2940-53. https://doi.org/10.1097/00007632-200011150-00017.

8. Du JP, Hao DJ, Fan Y, et al. Application of gelatin sponge impregnated with a mixture of 3 drugs to intraoperative nerve root block to promote early postoperative recovery of lumbar disc herniation. World Neurosurg. 2018; 114:e1168-73. https://doi.org/10.1016/j.wneu.2018.03.170.

9. Vos T, Allen C, Arora M, Barber RM, Bhutta ZA, Brown A, et al. Global, regional, and national incidence, prevalence, and years lived with disability for 310 diseases and injuries, 1990-2015: a systematic analysis for the global burden of disease study 2015. Lancet. 2016;388(10053):1545-602. https://doi. org/10.1016/50140-6736(16)31678-6.
10. Teng I, Han J, Phan K, Mobbs R. A meta-analysis comparing ALIF, PLIF, TLIF and LLIF. J Clin Neurosci. 2017:44:11-7. https://doi.org/10.1016/ij.jocn.2017. 06.013.

11. Resnick DK, Choudhri TF, Dailey AT, Groff MW, Khoo L, Matz PG, et al. Guidelines for the performance of fusion procedures for degenerative disease of the lumbar spine. Part 8: lumbar fusion for disc herniation and radiculopathy. J Neurosurg Spine. 2005;2(6):673-8. https://doi.org/10.3171/ spi.2005.2.6.0673.

12. Mobbs RJ, Phan K, Malham G, Seex K, Rao PJ. Lumbar interbody fusion: techniques, indications and comparison of interbody fusion options including PLIF, TLIF, MI-TLIF, OLIF/ATP, LLIF and ALIF. J Spine Surg. 2015;1(1): 2-18. https://doi.org/10.3978/j.issn.2414-469X.2015.10.05.

13. Harms J. True spondylolisthesis reduction and monosegmental fusion in spondylolisthesis. In: The textbook of spinal surgery; 1997. p. 1337-47.

14. Phan K, Rao PJ, Kam AC, Mobbs RJ. Minimally invasive versus open transforaminal lumbar interbody fusion for treatment of degenerative lumbar disease: systematic review and meta-analysis. Eur Spine J. 2015;24(5): 1017-30. https://doi.org/10.1007/s00586-015-3903-4.

15. Lan T, Hu SY, Zhang YT, Zheng YC, Zhang R, Shen Z, et al. Comparison between posterior lumbar interbody fusion and Transforaminal lumbar interbody fusion for the treatment of lumbar degenerative diseases: a systematic review and Meta-analysis. World Neurosurg. 2018;112:86-93. https://doi.org/10.1016/j.wneu.2018.01.021.

16. Foley KT, Holly LT, Schwender JD. Minimally invasive lumbar fusion. SPINE. 2003;28(15 Suppl):S26-35. https://doi.org/10.1097/01.BRS.0000076895.52418. $5 \mathrm{E}$.

17. Jenkins NW, Parrish JM, Mayo BC, Hrynewycz NM, Brundage TS, Mogilevsky $F A$, et al. The identification of risk factors for increased postoperative pain following minimally invasive transforaminal lumbar interbody fusion. Eur Spine J. 2020;29(6):1304-10. https://doi.org/10.1007/s00586-020-06344-4.

18. Fisher CG, Belanger L, Gofton EG, Umedaly HS, Noonan VK, Abramson C et al. Prospective randomized clinical trial comparing patient-controlled intravenous analgesia with patient-controlled epidural analgesia after lumbar spinal fusion. SPINE. 2003;28(8):739-43. https://doi.org/10.1097/01. BRS.0000058943.93281.28.

19. Hida T, Yukawa Y, Ito K, Machino M, Imagama S, Ishiguro N, et al. Intrathecal morphine for postoperative pain control after laminoplasty in patients with cervical spondylotic myelopathy. J Orthop Sci. 2016;21(4):425-30. https://doi. org/10.1016/j.jos.2016.03.004

20. Boulter JH, Curry BP, Szuflita NS, Miller CA, Spinelli J, Delaney JJ, et al. Protocolization of post-Transforaminal lumbar interbody fusion pain control with elimination of benzodiazepines and long-acting opioids. NEUROSURGERY. 2020;26(5):717-23. https://doi.org/10.1093/neuros/nyz232.

21. Prasartritha T, Kunakornsawat S, Tungsiripat R, Jampa J, Throngnumchai R. A prospective randomized trial comparing epidural morphine through intraoperatively placed epidural catheter and intravenous morphine in major lumbar spinal surgery. J Spinal Disord Tech. 2010;23(8):e43-6. https:// doi.org/10.1097/BSD.0b013e3181cd3048.

22. Berger CW, Crosby ET, Grodecki W. North American survey of the management of dural puncture occurring during labour analgesia. Can J Anaesth. 1998;45(2):110-4. https://doi.org/10.1007/BF03013247.

23. Tomov M, Tou K, Winkel R, Puffer R, Bydon M, Nassr A, et al. Does subcutaneous infiltration of liposomal bupivacaine following single-level Transforaminal lumbar interbody fusion surgery improve immediate postoperative pain control? Asian Spine J. 2018;12(1):85-93. https://doi.org/1 0.4184/asj.2018.12.1.85.

24. Du JP, Fan Y, Liu JJ, et al. Application of gelatin sponge impregnated with a mixture of 3 drugs to intraoperative nerve root block combined with robotassisted minimally invasive Transforaminal lumbar interbody fusion surgery in the treatment of adult degenerative scoliosis: a clinical observation including 96 patients. World Neurosurg. 2017;108:791-7. https://doi.org/10.1 016/j.wneu.2017.09.075.

25. Jun-Song $Y$, Kai-Xuan $L$, Lei $C$, et al. Cocktail treatment with a gelatin sponge impregnated with Ropivacaine, dexamethasone, and vitamin B12 promotes early postoperative recovery after percutaneous endoscopic lumbar discectomy: a retrospective, case-controlled study. Pain Physician. 2020;23(2):E211-8.

\section{Publisher's Note}

Springer Nature remains neutral with regard to jurisdictional claims in published maps and institutional affiliations. 